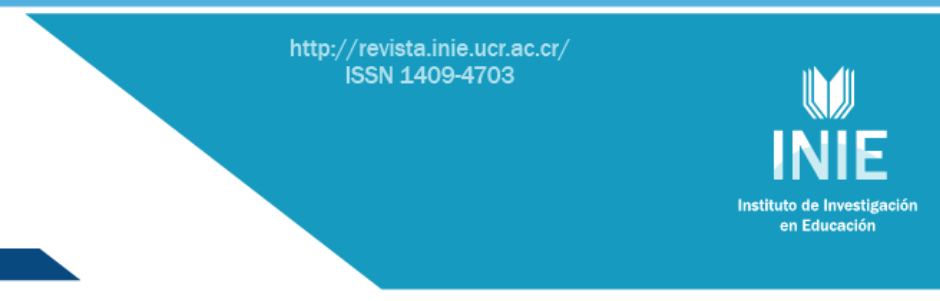

\title{
LA CONFIGURACIÓN DE LA CIUDADANÍA EN LOS PROGRAMAS DE ESTUDIO DE LA EDUCACIÓN CÍVICA AL INICIAR EL SIGLO XXI: EL CASO DEL ELEMENTO POLÍTICO DE LA CIUDADANÍA
}

THE CITIZENSHIP CONFIGURATION ON THE PROGRAMS' CIVIC EDUCATION STUDIES AT THE BEGINNING OF THE XXI CENTURY: THE CASE OF THE CITIZENSHIP'S POLITIC ELEMENT

Volumen 11, Número 2

pp. 1-30

Este número se publicó el 30 de agosto de 2011

César Toruño Arguedas

La revista está indexada en los directorios:

LATINDEX, REDALYC, IRESIE, CLASE, DIALNET, DOAJ, E-REVIST@S,

La revista está incluida en los sitios:

REDIE, RINACE, OEI, MAESTROTECA, PREAL, HUASCARAN, CLASCO 


\title{
LA CONFIGURACIÓN DE LA CIUDADANÍA EN LOS PROGRAMAS DE ESTUDIO DE LA EDUCACIÓN CÍVICA AL INICIAR EL SIGLO XXI: EL CASO DEL ELEMENTO POLÍTICO DE LA CIUDADANÍA \\ THE CITIZENSHIP CONFIGURATION ON THE PROGRAMS' CIVIC EDUCATION STUDIES AT THE BEGINNING OF THE XXI CENTURY: THE CASE OF THE CITIZENSHIP'S POLITIC ELEMENT
}

\section{César Toruño Arguedas ${ }^{1}$}

\begin{abstract}
Resumen: El artículo estudia la construcción del proyecto de ciudadanía a partir de un análisis de contenido del elemento político plasmado en los programas de estudio de Educación Cívica en el período 2000-2008. El proceso de selección de los contenidos, para lo cual se incluyó análisis de documentos y entrevistas a integrantes de las comisiones de reforma y revisión de los programas de estudio de la asignatura. A partir de una interrelación entre fundamentos curriculares, las circunstancias en que se desarrolla el proceso de selección de contenidos y su análisis crítico se concluye que la configuración de la ciudadanía en los programas de estudio de Educación Cívica no responde a criterios curriculares elementales, se desarrolla dentro de procesos coyunturales y marcado por redes, explícitas o no, de poder y subjetividades, todo lo cual determina la selección de contenidos y la construcción del proyecto de ciudadanía oficialmente establecida en los programas de estudio.
\end{abstract}

Palabras clave: EDUCACIÓN CÍVICA, CIUDADANÍA, CURRÍCULUM, PROGRAMAS DE ESTUDIO.

\begin{abstract}
The article examines of the citizenship project's construction from the content study of the political element depicted on the programs' civic education studies during the period 2000-2008. The selection process of the contents, for this, it was included documental analysis and interviews to the individuals that are part of the reform and review of the study programs' civic education. From the interrelation between curricular basis, the circumstances on which it is developed the selection process of contents and the critical analysis, it can be concluded that the citizenship's configuration on the programs on civic education studies does not answer to elemental curricular criteria. It is developed on in the context of the situation and marked by nets, explicit or not, of power and subjectivities. All this determines the content selection and the development of the citizenship's project officially established in the study's programs.
\end{abstract}

Key words: CIVIC EDUCATION, CITIZENSHIP, CURRÍCULUM, CONTENTS' STUDY.

\footnotetext{
${ }^{1}$ Máster en Planificación Curricular, de la Universidad de Costa Rica. Docente de Estudios Sociales, del Ministerio de Educación Pública, Costa Rica. Coordinador Proyecto Sortilegios Educativos.
}

Dirección electrónica: cesartoruno@gmail.com

Artículo recibido: 17 de enero, 2011

Aprobado: 28 de julio, 2011 


\section{Introducción}

Ante los retos culturales de la transnacionalización económica dentro del contexto de globalización y los problemas de legitimidad institucional dentro de la democracia costarricense, nuestro país ha profundizado, desde el año 2000, la discusión sobre la construcción de la ciudadanía y el papel de la educación como institución social impulsora y/o formadora de la ciudadanía.

Los ajustes educativos, en ejes transversales, contenidos y evaluación, realizados durante los últimos diez años, a los programas de estudio de la Educación Cívica, y al sistema educativo en general, son las muestras de la necesidad de un sistema educativo que responda a las situaciones sociopolíticas y económicas, coyunturales y estructurales, relacionadas con la construcción de la ciudadanía costarricense.

Las medidas impulsadas desde el Ministerio de Educación Pública (MEP) sobre el tema de ciudadanía se han caracterizado, muchas veces, por la construcción aislada de alternativas, como es el caso de los ejes transversales, la inclusión o eliminación de contenidos académicos, entre otras, sin que existan marcos reguladores para la selección cultural de contenidos, su organización, secuencia, alcance horizontal y la coherencia en relación con los fines de la educación costarricense, el perfil de salida de cada ciclo de secundaria.

Además del aislamiento entre cada medida, el Ministerio de Educación Pública ha planteado y ejecutado revisiones, reformas parciales o totales a los planes de estudio de Educación Cívica durante las últimas cuatro administraciones, Rodríguez Echeverría (19982002), Pacheco De la Espriella (2002-2006) y Arias Sánchez (2006-2010), lo cual podría interpretarse como la búsqueda de respuestas, parciales, a la formación ciudadana a partir de situaciones coyunturales del contexto social y de las políticas educativas de cada gobierno.

Por lo anterior, se impone la necesidad de realizar estudios sobre la construcción, explícita e implícita, de la ciudadanía como marco regulador generado dentro de un proyecto político-económico hegemónico con un impacto en los programas de estudio y, por ende, en los procesos formativos a nivel micro curricular. No obstante, la complejidad del tema impide un desarrollo integral en una sola investigación, razón por la cual, y como parte del proyecto de Investigación Aplicada para optar por el grado de Magíster en Planificación Curricular, el presente artículo realizará un análisis de la estructuración del elemento político de la ciudadanía en los programas de estudio de Educación Cívica de los años 2000, 2005 y 2008. 
Se ha escogido los contenidos de la categoría política de la ciudadanía en tanto que, la ciudadanía como construcción fundamental dentro del proyecto de democracia costarricense ha sido objeto de escrutinio público al iniciar el siglo XXI, principalmente en ésta categoría, ya que diversos actores sociales y políticos han reiterado la relación de fenómenos sociales con un debilitamiento en la construcción de la ciudadanía. Entre estos fenómenos estarían: el aumento del abstencionismo, la baja participación ciudadana, el cuestionamiento a la legitimidad de las instituciones democráticas, la desvalorización de la solución alternativa a conflictos, la distribución de la riqueza, la inclusión, los valores democráticos, entre otras.

Además, como bien afirma Giner (2007, p. 116), "Creer en la dignidad de la persona humana, en condiciones de modernidad, entraña ciudadanía", por lo que en sí, el tema ciudadanía tiene un valor agregado como escenario de reflexión sobre las diversas circunstancias que rodean a la Costa Rica de inicios del siglo XXI y los retos para la construcción de una sociedad más justa, solidaria, inclusiva y equitativa, donde se reivindique, en todos sus extremos, la dignidad de cada ser humano.

Para el análisis de los contenidos de del elemento político de la ciudadanía, se realizará un análisis de los criterios de selección y de organización de los mismos, los contenidos y los respectivos fundamentos curriculares que los configuran.

\section{Referencial teórico}

La educación, como institución social, se desarrolla en una interacción dialéctica con los procesos sociales, económicos, culturales y políticos, actualmente enmarcados en el proceso de globalización (Gimeno, 2001), tal premisa de la pedagogía crítica encuentra en las investigaciones y en las reflexiones de Freire y otros, el símbolo del inicio de una nueva corriente de investigaciones que, posteriormente, asumieron la denominación de Pedagogía Crítica $^{2}$, tendencia de investigación educativa que asume los postulados básicos de la teoría crítica de la Escuela de Frankfurt.

La pedagogía crítica ofrece alternativas metodológicas y análisis (Cabello, 2003) para visibilizar al sistema educativo como un escenario de conflicto sobre las diferentes formas de

${ }^{2}$ La denominación de pedagogía crítica es más una construcción anglosajona y extendida en Europa. No obstante, también puede recibir nombres como: Pedagogía Revolucionaria, Pedagogía de la Liberación, Pedagogía del Oprimido y otras. 
conocimiento y los roles docentes (Giroux y McLaren, 1998), relaciones pedagógicas que deben ser reconocidas como relaciones de poder y resistencias (Giroux y McLaren, 1998), no ideológicamente inocentes y donde los educadores pueden ser intelectuales transformadores (Giroux, 2006), dentro de un currículum oculto (Torres, 1998) y redes de poder que es preciso asumir y resistir desde una visión progresista.

En relación con el presente análisis, la pedagogía crítica reinterpreta la ciudadanía "en términos pedagógicos, como un proceso de regulación moral y de producción cultural, dentro del cual se estructuran subjetividades particulares en torno a lo que significa el hecho de ser miembro de un Estado Nacional' (Giroux, 2006, p. 23) y en cuyo proceso de selección y organización de contenidos confluyen distintas fuerzas en pro del establecimiento de una determinada visión de ciudadanía.

Tal definición nos conduce a profundizar la concepción de la ciudadanía desde una pedagogía crítica, ya que, en esta tendencia educativa, se reconoce al ciudadano como miembro de una democracia participativa y representativa, por lo que debe de fomentarse la conciencia sobre las responsabilidades del gobierno y el cogobierno (Cabello, 2003); también, implica la ruptura del discurso de la armonía incómoda (Giroux, 2006), que suaviza los conflictos cotidianos y estructurales en el sistema educativo.

Al mismo tiempo, nos exige una breve reconstrucción histórica de su configuración para el caso costarricense que para los objetivos del presente artículo, y dentro de la extensión de este, debe ser obviado; no obstante, es aconsejable una revisión de los estudios de la construcción de la ciudadanía costarricense en la segunda mitad del siglo XIX desde la visión del liberalismo (Molina y Palmer, 2000; Molina, 2002) y la utilización del sistema educativo como herramienta legitimadora de ese proyecto político mediante la creación de la Educación Cívica y los Estudios Sociales (véase: Solano, 2006; Palacios, 2006a, Palacios, 2006 b; Arias, Freer, Sole y Vásquez, 1998 y Vargas, 2001).

Asimismo, se debe contextualizar dentro de una serie de reformas a la formación ciudadana y la Educación Cívica llevada durante la última década en América Latina (Levinson y Berumen, 2007; Mejía y Perafán, 2006) y enmarcado dentro de un proyecto de ciudadanía neoliberal despolitizada (Jiménez, 1991), creadora de un consumidor que no necesita asociarse políticamente (Gimeno, 1998b), que establece la sociedad como la reunión de consumidores Lichstenztejn (1988), con derechos políticos restringidos sino que eliminados (Hindess, 2002); 
legitimador de un credo al mercado (Tamayo, 2003) con un sistema educativo como herramienta implementadora de ese proyecto (Torres, 2003).

Por último, se conceptualiza el currículum como "el proyecto selectivo de cultura, cultural, social, política y administrativamente condicionado, que rellena la actividad escolar, y que se hace realidad dentro de las condiciones de la escuela tal como se halla configurada" (Gimeno, 1998a, p. 40) que, desde la pedagogía crítica, se convierte en "problema moral" (Gimeno, 1998b) y herramienta vital para mantener o redistribuir las redes de poder entorno al capital cultural y su traducción en la desigual distribución de la riqueza; por lo cual no puede visualizarse como un producto neutral, objetivo y natural de los procesos sociales, ni exentos de conflictos socioculturales y político-económicos en los cuales diversos grupos de presión luchan por ampliar su posición, grupo dominante, o redistribuir pesos pedagógicos-sociales de sectores débiles y dominados.

En ese sentido, es necesario visibilizar las funciones de control (Apple, 1997) del currículum, y su papel históricamente configurado como escenario pedagógico crucial para la transmisión de un conocimiento oficial (Apple, 1996a) construido, y utilizado, por una ideología dominante, pero capaz de ser un espacio para la construcción de la emancipación (Kemmis, 1998).

\section{Metodología}

La investigación se sustentó en la tendencia de investigación Sociocrítica, ya que como establece Sandín (2003) se asume que la realidad es compartida, histórica, construida y dinámica, pero que existen redes de poder que pueden ser visibilizadas mediante el análisis de lenguaje y discurso (Kemmis, 1998, p. 119), lo cual era imperativo para la contextualización del diseño curricular.

Además, se asumió una posición epistemológica dominada por el construccionismo como elemento vital para devalar uno de los elementos de la interacción educativa para la construcción del significado: la selección y la organización de contenidos académicos, en el caso específico del elemento político de la ciudadanía. De igual modo, para la comprensión de este proceso, selección y organización de contenidos, se utilizó la teoría de investigación la teoría Crítica para establecer los supuestos de que: todo conocimiento está mediatizado por relaciones de poder, los hechos no pueden ser separados del campo de los valores y la 
ideología, el concepto o significado puede variar, pues está mediatizado por relaciones de producción capitalista y que determinados grupos son más privilegiados que otros.

Para el análisis de la selección y de la organización, como proceso y su producto plasmado en los programas de estudio, se utilizó el análisis de contenidos y entrevistas semi estructuradas.

En el caso del análisis del contenido, y siguiendo a Piñuel (2002), realizado a los programas de estudio es descriptivo-explicativo, ya que se realizó un análisis documental contextualizado desde un diseño vertical o intensivo, es decir, se ha reducido el total de material (tres programas de estudio), para realizar un análisis de palabras, relaciones entre conceptos, categorías, frases y oraciones.

El análisis utilizó un protocolo, es decir, una serie de parámetros que guiaron la recolección, distribución y análisis de los datos a recoger, el cual estableció la apertura de un libro de códigos y fichas de análisis. En el caso del libro de códigos, nos permitió, en primer lugar, conocer la naturaleza del documento (contenido, función, formas de interpretación, entre otros), en segundo lugar, se la creación de las categorías las cuales, para la investigación, fueron creadas previamente. La categoría involucró, como unidades de análisis, las unidades temáticas (búsqueda de conceptos y de corte mixto) y unidades lingüístico-proposicionales (búsqueda de oraciones y proposiciones, de corte cualitativo) para el análisis de los contenidos de los programas de estudio.

Las unidades de análisis fueron denominadas: Formas de Gobierno, Democracia, Valores de la Democracia, Democracia Costarricense, Formas de Participación, Marco Jurídico de la Democracia, Instituciones Democráticas, Sistema Electoral, Identidad de la Nación Costarricense, Otro y No es político. Por su parte, los diferentes niveles de secundaria (de sétimo a undécimo año) fueron numerados y se procederá a la ubicación de los contenidos por nivel para, posteriormente, realizar el análisis de cada contenido según las unidades temáticas y lingüístico-proposicionales.

Cada uno de los contenidos de los programas fue ubicado en las Unidades de Recepción paralelamente a la apertura y cierre de fichas de análisis, para lo cual se estableció como indicador el inicio y el cierre de cada período trimestral o la saturación de esta. En la última etapa, se procedió con la revisión del proceso de extracción de contenidos por unidades de análisis y al posterior análisis del mismo. 
También, se realizó el mismo procedimiento para el análisis de los fundamentos, explícitos o no, que determinaron la selección y la organización de contenidos.

Para el análisis del proceso de selección y organización de los contenidos, se entrevistará a tres exintegrantes de las comisiones, uno en el caso de la comisión 2008, a quienes se les realizó una entrevista semi estructurada que será transcrita y analizada mediante la apertura de Libro de análisis, donde cada informante fue enumerado, se crearon cuatro grandes unidades de análisis: información personal, información sobre el proceso, información sobre la selecciónorganización y recomendaciones.

Tomando en cuenta la información teórica previa y la entrevista semi estructurada se crearán subunidades de análisis para cada categoría y se procedió a ubicar la información dada por los informantes según las subunidades a las que pertenezcan, lo que dio paso a una revisión de la distribución de la información comparada con una lectura crítica-integral de cada entrevista para proceder al análisis integral de la información recopilada.

\section{Discusión de resultados}

La discusión de resultados se subdividirá en un análisis del proceso de reformas a los programas de estudio de Cívica entre el período 2000 y 2008 como mecanismo para entender el desarrollo de las reformas a los programas de estudio, los criterios de selección utilizados en las diferentes comisiones como eje para contextualizar y reinterpretar la construcción de los contenidos, la estructuración del elemento político de la ciudadanía en esos procesos y, por último, los fundamentos curriculares que influyeron en su selección.

\subsection{Proceso}

El proceso de revisión o reforma de los programas de estudio de Educación Cívica en el período 2000-2008, comentado por integrantes de las respectivas comisiones, nos permite, en primer término, reconocer la alta influencia del Ministro de Educación Pública y, por ende, de la administración de turno, no sólo como propulsor sino, además, como generador de criterios de selección para los contenidos, así como una notoria influencia y presiones de grupos políticos y económicos hacia la Comisión y el Consejo Superior de Educación para poder alterar los contenidos seleccionados y su organización, lo cual demuestra la fortaleza de un currículum 
oculto que, por su complejidad, supera las posibilidades de indagación y de discusión del presente artículo.

La influencia de sectores dominantes-políticos en un proceso técnico pedagógico es un marco contextual elemental en el análisis del diseño curricular y que debe ser asumido como un reto para la concreción de diseños más pertinentes a una realidad diversa en la que deben incluirse las diversas voces, tanto de sectores dominantes como de sectores débiles y los excluidos por el sistema.

Encima, resalta la incorporación a docentes en ejercicio casi de manera simbólica, pues rápidamente (dos semanas de iniciado el proceso) los docentes abandonaron la comisión, por diferentes motivos, y no existió remplazó para mantener esa "voz" docente dentro de la comisión y, al mismo tiempo, una débil fundamentación curricular del proceso, limitada a una charla inductiva para la comisión de reforma o revisión.

A lo anterior es necesario sumar el nulo proceso de sistematización de las experiencias de cada comisión, proceso que, de realizarse, podría enriquecer las labores de cada nueva comisión, ya sea con las experiencias exitosas del proceso o con las que, de alguna manera, pueden mejorarse.

El tiempo para realizar la reforma o la revisión se constituye en el elemento determinante en el trabajo de las comisiones, ya que los tres procesos se caracterizaron por realizarse en un lapso de 9 a 12 meses, iniciando normalmente en noviembre, pero con la agravante de que los contenidos debían estar seleccionados antes de marzo-mayo (los informantes no coinciden en el mes) por la presión de las casas editoriales para la elaboración de los libros de texto.

\subsection{Criterios de selección y organización de contenidos}

En relación con el proceso de selección y de organización de contenidos, los dos primeros procesos en 2000 y 2005, hubo un intento por concentrarse en una reforma de procedimientos más que de contenidos, en tanto que en el proceso del 2008 si existió la intención de una reforma total. Sin embargo, se ha podido determinar la inexistencia de criterios uniformados y curricularmente sustentados para la selección y la organización de contenidos, por lo que cada comisión diseña criterios emergentes marcados, notablemente, por la influencia del Ministro de Educación y por las subjetividades de los integrantes de cada comisión. 
Dentro de los criterios que pueden estructurarse a partir de la información dada por los entrevistados, se establece un débil marco teórico que sustente el proceso, una alta preocupación por diferenciar la Educación Cívica de Estudios Sociales (mediante los contenidos de cada asignatura) y para dotar de contenidos suficientes, a la Educación Cívica, para enfrentar la prueba nacional de bachillerato en la asignatura.

El contexto histórico es mencionado, implícitamente, como criterio de selección pero con la circunstancia de que es leído, e interpretado, por los miembros de la comisión, sin consultas profundas a sectores de la sociedad civil y, en particular, a docentes y a estudiantes, se genera una interpretación de las necesidades sociohistóricas a las que debe responder la Educación Cívica que, en el mejor de los casos, tiene un marcado sesgo.

Por su parte, el criterio de juicio de experto aunque determinante en los proceso $2000 \mathrm{y}$ 2005, no así en el proceso 2008, se limitó a la representación de una sola universidad (Universidad de Costa Rica) y a una sola escuela (Escuela de Historia) por lo que es necesario ampliar la participación de académicos de las otras universidades estatales y otras áreas académicas.

A nivel del criterio de la pertinencia, expresado en el proceso 2008, se trata de incorporar, los intereses del estudiantado como agente activo del proceso de enseñanza-aprendizaje y, al mismo tiempo, se reivindican los contenidos como método para abordar, o contrarrestar, la deserción presentada sobre todo en sétimo año. Aunque es un elemento innovador en los procesos y refrescante para el área, lo cierto es que se realizó sin la consulta a estudiantes y a docentes, por lo que vuelve a tener una visión desde los integrantes de la comisión, que puede ser no tan enriquecedor como en un escenario de consulta a esos sectores.

Para el caso de las coincidencias en las diferentes comisiones, los informantes señalan el peso de las editoriales en el proceso de selección y organización no sólo por el tiempo en que deben en que deben realizar el proceso para dar tiempo a la impresión de libros, sino, en la misma línea, como grupos de presión ante el cambio de contenidos, y la organización, pues dicho proceso les implicaría una inversión de recursos para actualizar sus libros de texto; reflejándose, como currículum oculto, los intereses eminentemente mercantiles de empresas educativas.

En el mismo sentido, de un currículum oculto, resulta revelador las referencias explícitas e implícitas a los intereses de sectores políticos-económicos para implementar un proyecto ideológico-cultural a través del currículum prescrito de secundaria, circunstancia que reivindica 
la importancia de determinante clarificar los criterios de selección para minimizar el impacto de esos intereses en los procesos educativos, al mismo tiempo que se impone la necesidad de reflexionar sobre esa influencia y el proyecto hegemónico que representa.

En relación con la organización de los contenidos, se mantiene la constante de la selección, es decir, un peso determinante a las subjetividades de los integrantes de la comisión. Por su parte, en los procesos 2000 y 2005 surge el criterio de organizar los contenidos cronológicamente y en los tres procesos impera, implícita y explícitamente, el criterio del nivel de desarrollo cognitivo según la teoría constructivista, aunque no es posible determinar la forma de aplicación de la misma.

Por último, las recomendaciones dadas por los informantes omiten la creación de lineamientos curriculares para la selección y la organización de contenidos, lo que podría ser producto de cierta cultura institucional de trabajo en los programas de estudio, es decir, puede existir un consenso no verbalizado de que los procesos de selección-organización de contenidos son emergentes y determinados por los integrantes de la comisión. No obstante, tal afirmación necesita un respaldo de una investigación a profundidad sobre las prácticas cotidianas del trabajo en esas comisiones, circunstancia que trasciende el objetivo de la presente investigación.

\subsection{Fundamentos}

Los fundamentos curriculares constituyen el referencial que permite el análisis, y la operacionalización curricular, de las circunstancias y de las demandas al sistema educativo que se expresan, explícita e implícitamente, en los procesos curriculares (principalmente en la creación de los perfiles de entrada y salida, la selección-organización de los contenidos y la recontextualización curricular del currículum prescrito en las aulas).

Para el presente artículo, se ha analizado la información de los siguientes fundamentos curriculares.

\subsubsection{Fundamento Filosófico}

El fundamento filosófico es uno de los elementos curriculares más complejos, ya que, además de manifestarse explícitamente en legislaciones y documentos oficiales, también se da 
en la construcción cotidiana de ese ser humano y ciudadanía, en la cual el contexto del proceso educativo puede sugerir la conformación, implícita, de un nuevo ideal de ser humano.

En tal sentido, el análisis del fundamento filosófico de la ciudadanía en los programas de estudio de Educación Cívica pasa por un análisis de los Fines de la Educación Costarricense, como gran conceptualización general del ideal de ser humano y ciudadanía, los perfiles de Salida de Tercer y Cuarto Ciclo en el área de Educación Cívica vigentes durante los procesos de reforma a los programas y, por último, los razonamientos explícitos en los programas de estudio de Educación Cívica de los años en estudio.

\section{a. Fines de la Educación Costarricense}

En 1957, como parte de la consolidación del proyecto hegemónico socialdemócrata impulsado por el Partido Liberación Nacional, se aprueba la Ley Fundamental de Educación, vigente hasta nuestros días, la cual refleja los fundamentos políticos, económicos y filosóficos socialdemócratas, partiendo de la conceptualización de la educación como un derecho humano (artículo 1 de la Ley Fundamental de Educación) capaz de fomentar el desarrollo de habilidades y destrezas en la ciudadanía necesarias para su incorporación social, cultural, política y económica en el Estado, y teniendo su mayor impacto en la concreción de los Fines de la Educación Costarricense.

La Ley Fundamental de Educación establece, en el artículo 2, que son Fines de la Educación Costarricense:

a) La formación de ciudadanos amantes de su Patria, conscientes de sus deberes, de sus derechos y de sus libertades fundamentales, con profundo sentido de responsabilidad y de respeto a la dignidad humana.

b) Contribuir al desenvolvimiento pleno de la personalidad humana.

c) Formar ciudadanos para una democracia en que se concilien los intereses del individuo con los de la comunidad.

d) Estimular el desarrollo de la solidaridad y de la comprensión humana.

e) Conservar y ampliar la herencia cultural, impartiendo conocimientos sobre la historia del hombre, las grandes obras de la literatura y los conceptos filosóficos fundamentales. (Asamblea Legislativa de Costa Rica, 2007b) 
En ninguno de los procesos de reforma curricular fue explícito el uso de los Fines de la Educación Costarricense como fundamento filosófico para el proceso de selección y organización de contenidos.

Asumiendo el papel implícito de los fines, tendríamos que reconocer que, en primer lugar, el inciso "a" es un mecanismo de selección y organización implícito en las propuestas de programas de estudio realizadas en el año 2000, 2005 y 2008 ${ }^{3}$, para el eje de Identidad Nacional con un énfasis en la pertenencia a la institución, la comunidad y la nación costarricense, relacionando ésta última con símbolos, tradiciones, costumbres y otros.

Asimismo, las comisiones organizan dichos contenidos en sétimo año (2000 y 2005) y octavo año (2008), asumiendo, implícitamente, la importancia de acercar al estudiante a un "amor por la patria" paulatinamente, estableciendo relaciones de lo micro a lo macro y en una organización temporal antes de iniciar el estudio de derechos humanos, sistemas políticos y democracia costarricense.

El inciso "c" es el elemento del fundamento filosófico determinante en las reformas a los programas aunque, igual que los demás elementos, en forma implícita y concentrando en el área de la democracia, ya que las diferentes comisiones prestan alta atención al tema sin embargo, hay diferencias notables sobre el abordaje del área, mientras que en las reformas de los años 2000 y 2005 no se conceptualiza la democracia, en la reforma del año 2008 si se da una primera conceptualización en noveno año, con claras referencias históricas la formación de derechos y la estructura actual de la democracia, entiéndase, representación-participación, igualdad de trato (tocando aspectos económicos que van en relación con lo establecido en el inciso c de los Fines de la Educación), derecho de manifestación y condiciones básicas, derecho de elegir y ser electo, legitimidad y diversidad de fuentes de información.

Es importante resaltar que, en el caso de la caracterización de la democracia costarricense, existe una mayor amplitud en los programas de estudio. No obstante, existen diferencias notables en el enfoque de las Comisiones 2000, 2005 y 2008, ya que, mientras en los programas 2000 y 2005 se da una caracterización más conservadora y limitada ${ }^{5}$, en los

3 En el proceso del 2005 no se dio selección ni organización de contenidos en el elemento político.

4 El inciso "b" tiene una relación con el fundamento psicológico que se analizará posteriormente.

5 Incluye solamente las características presidencialismo, división de poderes, constitucionalismo y centralismo. 
programas 2008 se da un enfoque más amplio y profundo, ${ }^{6}$ lo cual podría interpretarse como un proceso lógico de formación conceptual para formar una ciudadanía en un momento coyuntural de crisis y de cuestionamientos.

La anterior situación también es evidente en el área de los valores de la democracia, donde ambas propuestas dedican un espacio a la formación de los valores considerados como esenciales en la democracia, existiendo una notable diferencia entre las propuestas de 2000, 2005 y 2008, ya que en las dos primeras de estas se hace referencia a valores ${ }^{7}$ dentro de lo que podría interpretarse una concepción más limitada de democracia, ${ }^{8}$ en tanto que la reforma 2008 amplía la gama de valores ${ }^{9}$ no sólo conceptualizándola como espacio de interacción sino, además, como un espacio legítimo donde se pueden resolver conflictos y donde la diversidad debe ser un eje base, atendida con respeto y, en caso de desacuerdos, atendida por los canales de la institucionalidad democrática.

Además, se da una relación explícita entre Democracia y formas de gobierno apostando por una caracterización ideológica, pragmática e histórica de las diferentes formas de gobierno y la legitimación de la democracia como forma de gobierno y de sus instituciones ${ }^{10}$.

La diferencia entre las propuestas 2000, 2005 y 2008 es en la aparición de la caracterización de diferentes ideologías dentro de la democracia para la reforma del 2008 y el

6 Es caracterizado por una cultura Democrática (incluye gradualismo, participación, deliberación, negociación, legalidad y comunicación política), organización institucional (división de poderes, constitucionalismo y presidencialismo), mecanismos de incidencia (cartas, reuniones, presentación de propuestas), mecanismos de rendición de cuentas, abolición del ejército, valores democráticos (descritos en el punto 3) y desafíos de la cultura política ( fortalecimiento de valores y prácticas democráticas, tolerancia vrs intolerancia, propaganda y comunicación educativa)

7 Incluye los valores de la tolerancia, la igualdad, el pluralismo, la responsabilidad, el uso racional de la libertad, el respeto por la vida y la persona humana, la solidaridad y la paz. En sétimo año, primer trimestre, se da el valor del respecto étnico y la inclusión.

8 No puede establecerse la amplitud o no del concepto de democracia utilizado por la comisión en tanto no existen registros de ese proceso y no quedo una visión explícita en los programas.

9 Se impulsa los valores de: libertad, igualdad política, solidaridad y equidad, tolerancia y respeto, paz, honestidad. Así mismo, se desarrollan actitudes como respeto a la libertad, fortalecimiento de la libertad individual y colectiva, respeto por la ley y las normas, búsqueda de la igualdad política, social y económica, generosidad-cooperación, derecho al desacuerdo, aprecio al gradualismo, a los espacios de participación y transparencia, respeto hacia las mayorías y minorías, respeto a la institucionalidad democrática y aprecio por el diálogo y la negociación

10 Todas las propuestas coinciden es destacar el papel de las instituciones del Estado como forma de participación y de vivencia democrática siendo más extenso el abordaje realizado por las propuestas 2000 y 2005 con las siguientes instituciones: Sala Constitucional, Defensoría de los Habitantes, Ministerio de Trabajo, Tribunales de Justicia, Caja Costarricense del Seguro Social, Oficina de Protección al Consumidor, Patronato de la Infancia, Comisión Nacional de Asuntos Indígenas, Gobiernos Locales, las instituciones privadas, el Tribunal Supremo de Elecciones, para finalizar en undécimo año con: la División de poderes, Ministerios, Contraloría General de la República, Defensoría de los Habitantes, Corte Suprema de Justicia, Ministerio Público, Defensa Pública, Organismo de Investigación Judicial, Municipalidades, Instituciones Autónomas, Tribunal Supremo de Elecciones y Partidos Políticos. 
ordenamiento, en este último punto es importante destacar que en el año 2000 y 2005 se propuso como contenido por desarrollar en undécimo año, mientras que en la reforma del 2008 se ubica en décimo año, siendo una muestra de que durante el último proceso se dio una selección de contenidos marcado por el abordaje de la democracia a tal punto que los contenidos se extenderán desde noveno hasta undécimo año.

Por último, el perfil de ciudadanía establecido en los programas de estudio se encuentra marcado por el tema de la participación, los programas concuerdan en dar una importancia a la participación electoral, generando un acercamiento con los procesos electorales de la institución, en el caso de las propuestas de 2000 y 2005 en sétimo y noveno año (con un breve bloque de contenidos en undécimo año) y el programa de estudio 2008 con un abordaje en noveno y décimo año ${ }^{11}$.

También se hace referencia a los diferentes mecanismos de participación, distribuidas en tercer ciclo para las propuestas 2000-2005 y noveno y décimo para la propuesta 2008. En el caso del programa de estudio del año 2008, sus contenidos en el área de la participación, dibujan el perfil de una ciudadanía altamente comprometida con la "solución a la tica" de los conflictos, es decir, una solución negociada, pacífica, institucional y respetando las leyes y autoridades, lo cual es coherente con las preocupaciones de sectores dominantes ante las manifestaciones sociales ocurridas desde el 2000 (Combo ICE) y hasta el 2007 (Referendo del TLC con Estados Unidos).

Por último, en el área de democracia, se perfila una ciudadanía conocedora de la legislación general que enmarca sus derechos, aunque se da descontextualizadamente y desde una visión muy jurídica de su ejercicio, así como una relación el Estado como garante de los derechos, institución social e históricamente configurada, manteniendo como eje de comparación el Estado con la Polis griega.

En síntesis, la aplicación implícita del inciso "c" de los Fines de la Educación Costarricense, como delimitador del perfil de la ciudadanía demandada por el espacio filosófico, muestra una tendiente preocupación del Ministerio de Educación Pública por legitimar los espacios e instituciones de la democracia, así como posicionarla como una institución

11 El tema será retomado en décimo año, con referencias a la importancia del sistema electoral en Costa Rica, papel del Tribunal Supremo de Elecciones (funciones), tipos de elecciones, partidos políticos (estructura, financiamiento, ideologías, participación y sufragio, abstencionismo, comunicación política (propaganda, discursos, agendas políticas). 
perfectible. En ese mismo sentido, aporta una respuesta institucional a la denominada crisis de gobernabilidad-social caracterizada por las diferentes formas de descontento manifestadas por sectores populares ante el funcionamiento de la democracia costarricense en temas como el Combo ICE y el TLC con Estados Unidos.

Por último, en los tres programas se establece una relación, implícita, entre el inciso "d" de los Fines de la Educación, "Estimular el desarrollo de la solidaridad y de la comprensión humanas" y los valores. En el caso de los programas 2000 y 2005 se da con la inclusión de valores como la igualdad, el respeto por la vida y la persona humana y la solidaridad; por su parte, en el programa 2008 se impulsan los valores de solidaridad y equidad (una innovación que establece una relación más directa con el inciso "d" ) y actitudes como la búsqueda de la igualdad política, social y económica y generosidad y cooperación, en cambio en los programas 2008 se establece una débil referencia en el área de valores al citar la solidaridad y equidad.

\section{b. Perfil de salida del estudiante en el III y IV ciclo en el área de Educación Cívica}

El perfil de salida es la manifestación explícita más importante, en el currículum prescrito, de las intencionalidades del proyecto educativo oficial, en este se establecen los mínimos deseables por la estructura oficial en relación con el perfil ontológico de ser humano y ciudadanía.

En tal sentido, en los programas 2000 y 2005, como construcción pedagógica dentro de una lógica oficial uniforme, enmarcada políticamente por la continuación de dos administraciones del Partido Unidad Social Cristiana, mantienen un mismo perfil de salida para III y IV ciclo en el área de Educación Cívica, en tanto el programa 2008 ofrece un perfil nuevo enmarcado por el ascenso de un gobierno liberacionista y el impulso del Ministro de Educación al proyecto "Ética, estética y Ciudadanía" como reconceptulización y operacionalización de la formación ciudadana. Por tales circunstancias, el análisis de los perfiles de salida se realizará categorizando el perfil del programa 2000 y del 2005 (unificados) y el programa 2008.

\section{- Perfil de Salida en los Programas de 2000 y de 2005}

Como puede apreciarse en la Tabla No. 1, el Perfil de Salida III Ciclo vigente para los programas 2000 y 2005 divide, implícitamente, la ciudadanía en tres grandes áreas: 
a). Escenario jurídico: se establece la vinculación con las responsabilidades y derechos del individuo, lo valores éticos y los Derechos Humanos, marco jurídico de derechos y deberes, con énfasis en leyes de Igualdad Real de la Mujer y protección a niñez.

b). Vivencia de participación: altamente vinculada con la participación electoral, en principio ejercida dentro de la institución, pero con ejes expansivos a la participación en procesos nacionales, y con el agregado de ejercer una colaboración en dichos procesos. También, se visualiza como participante en las actividades cívicas (actos cívicos) de la institución y la comunidad,

c). Lugar de interacción: estableciendo, en primer lugar, una relación como sujeto integrante de la nación costarricense, asimismo, se apuesta por la formación para la solución pacífica de los conflictos, espíritu de servicio familiar, social y comunal, respeto y tolerancia a las opiniones, criticidad ante las irregularidades y manejos incorrectos de fondos públicos y, por último, como consumidor y sujeto que contribuye al progreso del país mediante el pago de impuestos.

En el caso del área jurídica, la idealización del ser humano y de ciudadanía asocia el ejercicio de la ciudadanía en relación con el conocimiento de los principios legales que regulan la democracia, por lo cual es un área abordada desde la lógica de la democracia formal, en ese misma interpretación se desarrolla el tema de la participación limitada a aspectos electorales y de asistencia a actos oficiales.

Por su parte, la propuesta en el área de interacción es donde más claramente se esboza un proyecto de formación ciudadana dirigido a inculcar la participación en los conflictos con la "solución a la tica" con características de la solución pacífica de conflictos, espíritu de servicio y, al mismo tiempo, trata de responder a la preocupación social por el tema de la corrupción y la preocupación política por una mejora en el cobro de los tributos. No obstante, resalta la incursión de los principios neoliberales al establecer el elemento de formación del consumidor, lo cual es un claro indicio de la reconfiguración de los fundamentos curriculares en relación con el proyecto neoliberal.

En el caso del perfil de salida de la educación diversificada, se da un seguimiento de las tres categorías implícitas formuladas para Tercer Ciclo, pero con un elemento innovador: la incorporación de una categoría laboral. Tal inclusión, aunque leve dentro de los doce puntos del perfil de salida, remite a la importancia de la formación ciudadana de la educación diversificada dentro de una antesala, o preparación, para la inclusión de la futura ciudadanía dentro del sistema productivo. 
En la categoría Jurídica es notable la disminución de elementos del perfil de Tercer Ciclo a Diversificada, de seis a dos, lo cual es lógico dentro de una conceptualización de la Educación Diversificada como consolidación y profundización del perfil de salida del Tercer Ciclo, en este caso con un énfasis en una ciudadanía que asuma responsabilidades, en términos del perfil "sin excepción", en el ejercicio de sus derechos y deberes.

Además, establece una relación entre el respeto al marco jurídico y las normas y una "sana y edificante convivencia social", siendo un claro reflejo de una conceptualización de una ciudadanía ideal integrada en una polis social y política donde sus integrantes tengan, como marco común de interacción, el respeto por los marcos jurídicos sin establecerse, como si se hace en relación con la información, la necesidad de un criterio crítico sobre estos.

La categoría de Escenario de Convivencia refleja, en consonancia con el perfil del Tercer Ciclo, una concepción de una ciudadanía muy limitante en el área de la participación; establece un impulso al interés por la participación en asuntos públicos comunales y nacionales (profundización de la propuesta en el Tercer Ciclo) sin una clara explicitación de los escenarios de participación y la criticidad ante los mismos.

También, se establece la participación en organizaciones privadas y estatales de bien social, éste último concepto presenta una gran ambigüedad y la referencia en general omite la participación en otras organizaciones como No Gubernamentales y organizaciones privadas con un carácter diferente al asistencialismo asignado dentro de la conceptualización de "bien social".

Por último, existe una "anuencia" a la participación femenina, concepto que en sí representa debilidades en la concepción de género y la participación política y laboral, sin establecer referencias explícitas a una criticidad al estado patriarcal y el machismo y, aún más preocupante, se omiten referencias a otras minorías de origen sexual, étnico y cultural que han sufrido discriminaciones en sus intentos de participación en diferentes aspectos de la estructura social y la estructura de poder.

Como escenario de interacción, se denota la continuidad, con respecto al perfil de salida del Tercer Ciclo, de una correlación entre la interacción y la formación de una ciudadanía con un conocimiento de la identidad nacional, lo cual responde a la lógica de preservación de lazos mínimos de reconocimiento mutuo, de un origen común, para mantener el Estado Nacional dentro de la interacción en épocas de globalización.

Igual situación, de continuidad, se da con la formación de la legitimidad e importancia del uso de los mecanismos de resolución pacífica de los conflictos, tema crucial dentro del contexto 
de agitación social y política durante, y post, movimiento social conocido como Combo ICE. EI fomento de esa legitimidad iría dentro de la construcción del imaginario colectivo de una "solución a la tica", donde la violencia y la ruptura con la institucionalidad son antivalores que deben ser rechazados por ir contra nuestra supuesta identidad.

La inclusión del respeto por la diversidad cultural (etnia, género, credo religioso, ideología y nacionalidad) representa un primer paso en la aceptación de una sociedad costarricense cada vez más diversa; sin embargo, destaca su silencio tácito al tema de la diversidad sexual lo cual reitera lo limitado de la conceptualización implícita de la democracia y sus valores y, por tanto, de la proyección de ciudadanía esperada. En ese sentido, la inclusión de la criticidad "ante la situación actual del país, el rescate de los valores éticos" son elementos difusos que, ante su ambigüedad, dificulta su operacionalización y evaluación en el currículum.

Por último, la introducción de la "habilidad de selección y uso correcto de la información" y "juicio crítico ante la información que dan a conocer los medios masivos de comunicación" asumen la responsabilidad de formar una ciudadanía para interactuar en una polis cada vez más mediatizada por los grandes corporaciones de información y por intentos, cada vez más sistematizados, de la incorporación de medios alternativos, principalmente en la web, aunque tal perfil sólo se enfoca en la información como producto dirigido y no en los valores morales, visiones ideológicas y agendas de las industrias mediáticas dirigidas al entretenimiento, por citar un ejemplo.

\section{- Perfil de Salida Programa 2008}

Dentro de la reconceptualización de la ciudadanía y su traducción en la reestructuración del programa de estudio, la reforma al programa de Educación Cívica estableció un nuevo perfil de salida para Tercer Ciclo y Educación Diversificada, que refleja la búsqueda por "desarrollar los conocimientos, valores, actitudes, prácticas, competencias, en dos niveles: uno al final de noveno y otro al final de undécimo" (MEP, 2008. p. 31), estableciendo, explícitamente, la profundización, en el nivel de diversificada, de las habilidades establecidas para tercer ciclo.

El perfil de salida establecido es el de una persona ciudadana crítica e integrada, delimitando dichos conceptos con las siguientes especificaciones del perfil (MEP, 2008, pp. 31 $32)$; 


\section{Crítica:}

- Busca informarse y formarse criterio sobre los asuntos colectivos y públicos.

- Sabe expresar sus opiniones críticas y ponderar otras opiniones.

- Hace respetar sus derechos y los de las demás personas ciudadanas y habitantes.

- Posee competencias ciudadanas.

- Demanda una democracia más profunda y sostenible.

- Más inclusiva, con mejores instituciones y con una cultura política más democrática.

Integrada:

- Su sentido crítico no la lleva a disociarse.

- Ante la insatisfacción, crítica o pérdida de apoyo institucional busca la incidencia individual o colectiva, para mejorar aquello que lo requiera.

- Conoce los mecanismos institucionales y legales de incidencia.

- Participa cuando lo considera importante en forma directa o a través de sus representantes.

(MEP, 2008, p. 31)

Es destacable que, a diferencia de los programas 2000 y 2005, el programa establece macrocategorías de dos ejes para integrar elementos con una amplitud de interpretación que sólo puede ser atendida dentro del contexto explícito del programa.

En la categoría Crítica, los primeros tres elementos establecen una relación lógica dentro del desarrollo de los programas de Educación Cívica, y por ende la interpretación oficial y convencional de ciudadanía: búsqueda de información, formación de criterio, expresión y escucha de opiniones, defensa del marco jurídico de la ciudadanía, centrado en los derechos y deberes; todo enmarcado por la relevancia, implícita, del reconocimiento de la cosa pública y la identificación en la polis moderna como sustentos de la interacción ciudadana.

Sin embargo, el cuarto elemento, establece competencias ciudadanas las cuales incluyen la deliberación, la negociación, la participación-representación y la comunicación social (MEP, 2008, pp. 32-34), que se subdividen para especificar el perfil de salida.

La especificación de las competencias ciudadanas refleja la preocupación implícita ante un contexto de la sociedad costarricense marcada por el aumento del abstencionismo (elecciones 1998, 2002 y 2006), las protestas sociales y la inherente ruptura con la "solución a 
la tica" (siendo referentes claves protestas contra el Combo ICE y TLC con los Estados Unidos) y el aumento, reflejado en las encuestas, de la desconfianza y la crítica contra instituciones baluartes de la democracia costarricense.

En tal sentido, los objetivos de las competencias de deliberación, negociación, generación de consensos y manejo de disensos y participación-representación son una profundización y sistematización más estructurada del marco propuesto en los programas 2000 y 2005, dirigidos a generar una ciudadanía comprometida con el sistema democrático, legitimante de éste y sus instituciones, con destrezas y habilidades para la interacción en la polis costarricense, crítico y activo, pero sin romper con la institucionalidad, los ejes democráticos ni desasociarse de la polis y sus conciudadanos.

Resulta innovador, frente a los programas 2000 y 2005, la inclusión de la Comunicación Social y Política como competencia a desarrollar, pues es una aceptación del reto social, político y cultural, en la formación ciudadana, generado por la era de la información. Tal incursión reconoce a los medios de comunicación como formadores de opinión ante los cuales la ciudadanía debe tener un criterio crítico, tema que tomó relevancia durante la discusión del TLC con los Estados Unidos, principalmente en el referendo.

El último elemento de la categoría Critica establece la demanda de una democracia más profunda y sostenible, con los criterios de ser más inclusiva, con mejores instituciones y con una cultura política más democrática, lo que implica una profundización del inciso "a" y "c" de los Fines de la Educación los cuales, en forma implícita, son los fundamentos claves en la propuesta de la categoría Crítica del perfil de salida.

Por su parte, los cinco elementos de la categoría Integrada del perfil de salida establecen una relación directa con la preocupación gubernamental y de ciertos grupos dominantes por la "ingobernabilidad" y la ruptura con la "solución a la tica" demostrada en las diferentes formas de resistencias entre el año 2000 y 2007 (lucha del Combo ICE y proceso de discusión del TLC con los Estados Unidos).

Se manifiesta una clara preocupación por la formación de una ciudadanía que no esté disociada de su "polís" y su estructura social, que asuma protagonismos en la solución de sus insatisfacciones, conocedor de los mecanismos institucionales y legales de incidencia.

Ahora bien, la comprensión ontológica de la ciudadanía esbozada en los perfiles de salida de los programas de estudio 2000, 2005 y 2008 necesita, para una visión integral del currículum prescrito, el análisis de especificaciones y concepciones dadas en los documentos oficiales, 
para lo cual realizaremos un análisis de los fundamentos, explícitos o no, de la ciudadanía establecidos en estos programas de estudio de Educación Cívica y los acuerdos del Consejo Superior de Educación que impulsan cada reforma a los programas.

\subsubsection{Fundamento Económico}

Al iniciar la década de los ochenta toman mayor fuerza, en Costa Rica y América Latina en general, las voces internas y externas, en el primer caso principalmente jóvenes intelectuales y políticos no alineados con el proyecto económico socialdemócrata defendido por Figueres Ferrer y Daniel Oduber y, en el segundo caso, liderado por el Fondo Monetario Internacional y el Banco Mundial, que claman por una sustitución del viejo modelo económico y político socialdemócrata por un nuevo modelo neoliberal. Sin embargo, en el caso costarricense no será hasta 1985 cuando se inicie la profundización del modelo Neoliberal (Vargas, 2002) no sólo en aspectos económicos sino, además, en aspectos institucionales y culturales.

Como institución con un rol cultural determinante, el Ministerio de Educación Pública (MEP) inicia una serie de transformaciones para adaptarse a las exigencias socioculturales de Costa Rica y el proyecto neoliberal, siendo el primer escenario el de las reformas al presupuesto, caracterizadas por la disminución de la inversión en educación (Korten, 1997; para el caso de la educación superior se puede ver Pérez,1992; Pérez y González, 1991), en segundo lugar se dan reformas institucionales como la creación de Colegios Científicos, Experimentales Bilingües y el impulso a los Colegios Técnicos, entre otras reformas para ajustar al MEP a la demanda de creación de mano de obra especializada; así como un replanteamiento de contenidos, formas de enseñanza y tiempos educativos (principalmente horas por asignatura y días lectivos) con el objetivo de mejorar la eficiencia y eficacia del proceso educativo.

En tal escenario, de reformas en el sistema educativo enmarcadas por el proyecto neoliberal, el currículum costarricense asumió un nuevo fundamento económico: una mezcla de socialdemócrata y neoliberal, el primero dominante en los sistemas jurídicos de la educación, como la Ley Fundamental de Educación, y el segundo impulsor, implícito y explícitamente, en la selección de contenidos, creación e impulso de nuevas modalidades educativas y asignaturas y otras áreas del sistema educativo, estratégicas para la formación de una mano de obra requerida por un país en proceso de transnacionalización. 
En tal sentido, durante las últimas dos décadas se realizaron reformas en los programas de estudio de Educación Cívica que, paulatinamente, denotan más explícitamente la preocupación por la formación de una ciudadanía altamente concentrada en la formación de destrezas, habilidades y conocimientos que le permitiesen desenvolverse en una sociedad de mercado, principalmente como trabajador y consumidor, por tal motivo, en la reforma del año 2000 se incorpora el tema de los derechos-responsabilidades del trabajador, valores del trabajo, los derechos del consumidor y globalización, los cuales abarcan gran parte del temario del décimo año, nivel estratégico en tanto es el año previo a la conclusión de estudios en secundaria y, por ende, de la inserción laboral del ciudadano.

En la reforma del año 2005, no se dan cambios en los contenidos determinantes y en área de los contenidos relacionados con la formación de mano de obra y consumidores, se mantienen la selección y la organización realizada en el año 2000. En el caso de la reforma del 2008 "Ética, Estética y Ciudadanía" sí se da una transformación en contenidos importantes, en tanto que el tema de derechos y de deberes laborales, legislación laboral, derechos del consumidor y otras relacionadas con el área productiva son reducidas a un trimestre de undécimo año, situación que demarca la disminución en su importancia estratégica dentro del diseño curricular en tanto que, para efector prácticos, undécimo año tiene sólo dos trimestres (por la aplicación de la prueba Nacional de Bachillerato) lo que conlleva una de serie de circunstancias pedagógicas que atentan contra la eficiencia y la eficacia en la transmisiónconstrucción de esos conocimientos, ya que, en general, el cuerpo docente centrará sus esfuerzos en un estudio superficial de los temas para iniciar los repasos de contenidos para la Prueba Nacional.

Sin embargo, el fundamento económico neoliberal no deja de ser un peso importante en el diseño curricular en la reforma del 2008, pues el propio Ministro de Educación Pública, en la presentación de los nuevos programas de Educación Cívica, justifica la importancia de la reforma en tanto;

Son industrias [entretenimiento] en las que el arte y la alta tecnología se dan la mano para generar mejores empleos y las mayores rentabilidades. Pero eso no es cierto solamente de las industrias del entretenimiento; también ocurre lo en las industrias electrónicas (...). El sector servicios, que se expande rápidamente en nuestros países, es otro ejemplo de la importancia que tienen la sensibilidad y las destrezas o competencias que da una buena educación ética, estética y ciudadana: los hoteles, los hospitales, los bancos, el comercio, 
todos dependen claramente del atractivo y el buen trato que ofrecen a sus clientes y, por supuesto, a sus propios funcionarios (Ministerio de Educación Pública, 2008, p. 10).

Por lo tanto, el fundamento económico neoliberal en la reforma del 2008 es más sutil, pero sin dejar de ser un marco regulador que, para el caso de los programas de Educación Cívica, pierde ponderancia explícita ${ }^{12}$ frente al contexto sociocultural y la necesidad de ofrecer una respuesta pedagógica a circunstancias como el aumento del abstencionismo y la manifestación de resistencias y oposiciones fuera de la "solución a la tica".

\subsubsection{Fundamento sociocultural y político}

El sistema educativo, como institución cultural, asume implícita o explícitamente la premisa de que hay aspectos "de la cultura del grupo, que no tendrán lugar de forma satisfactoria, o que no se producirán en absoluto, a no ser que se suministre una ayuda específica, que se pongan en marcha actividades de enseñanza especialmente pensadas con este fin" (Coll, 1991, p. 30).

La anterior premisa refleja la importancia del fundamento sociocultural, entendido como la extracción de una serie de necesidades y aspiraciones de la sociedad e interpretado por los grupos dominantes y con control de la estructura oficial educativa, ha sido, históricamente, el gran configurador de la formación ciudadana como proyecto político y social originado desde el surgimiento de los Estados Nacionales europeos; el fundamento político, por su parte, es la influencia del anhelo de ciudadano, polis y gobierno que se impulsa desde los sectores dominantes, por tal razón y encontrando puntos comunes entre estos fundamentos, se procederá a realizar el análisis de los dos fundamentos como unidad en interacción dialéctica.

En el caso costarricense, el primer gran movimiento explícito de correlación entre un proyecto político hegemónico y la organización de la construcción de la ciudadanía en el sistema educativo se da en 1886 con la reforma educativa y la aparición de la Educación Cívica como una asignatura como procesos lógicos dentro del ideal liberal, gobernante y dominante en

\footnotetext{
12 A nivel implícito, la reforma del 2008 se enmarca dentro del proyecto Ética, estética y ciudadanía el cual puede ser interpretado como una reconceptualización de la ciudadanía siempre dentro de la concepción neoliberal y su función productiva. Para profundizar dicho debate, se recomienda Toruño (2010).
} 
la época, de construir una sociedad y ciudadanía apegado a los principios y a los anhelos liberales.

En los años recientes, las últimas cuatro administraciones han realizado cambios de forma y de fondo a los programas de Educación Cívica siempre como respuesta pedagógica a demandas sociales, culturales, políticas y económicas del momento histórica en que se hizo la reforma. De tal forma, en los programas 2000 y 2005 se explicita esta relación al manifestarse que

Los cambios sociales, económicos, culturales, científicos, ambientales y tecnológicos del mundo contemporáneo, han exigido al currículo educativo no solo aportar conocimientos e información, sino también favorecer el desarrollo de valores, actitudes, habilidades y destrezas que apunten al mejoramiento de la calidad de vida de las personas y de las sociedades. (MEP, 2000 , p. 4; MEP, 2005, p. 5)

Dentro de esos cambios, implícitamente reconocibles, se establece la formación de una ciudadanía que tendrá que jugar un rol activo en los procesos de producción, principalmente como trabajadores, y como seres humanos dentro del fenómeno de la globalización en sus diferentes manifestaciones. ${ }^{13}$

Por su parte, el programa 2008 es sumamente explícito a la hora de describir el marco sociocultural al cual responde la formación ciudadana, puesto que establece, y explica una Costa Rica afectada por violencia, desigualdad, problemas de representación, desconfianza, fragmentación de la identidad (MEP, 2008, pp. 28-29).

Por lo tanto, el elemento político de la ciudadanía responde a una crisis de legitimidad (problemas de representación y desconfianza en la institucionalidad estatal), ruptura de relaciones sociales (desconfianza punto II y fragmentación de la identidad).

Estamos pues, ante la respuesta pedagógica más sistematizada y compleja elaborada para la formación ciudadana desde inicio del siglo XXI, incluso, si tomamos en cuenta los contenidos y su estructuración, prácticamente responde al diagnóstico realizado por Luis Paulino Vargas (2001) sobre la crisis de la sociedad costarricense.

Además, se visualiza fundamento político de los programas 2000, 2005 y 2008 con la constante de romper con la concepción de democracia representativa, rescatando la

13 Ambos temas se impulsan en los contenidos de $10^{\circ}$ año, concentrando hasta un $80 \%$ del temario total del año. 
participación activa y la paulatina ampliación del concepto de democracia a una democracia deliberativa, inclusiva, solidaria y equitativa, sin que esto menosprecie el alto papel legitimante de la institucionalidad democrática y la búsqueda por incluir a los estudiantes dentro de la misma.

\section{Conclusiones}

La configuración del elemento político de la ciudadanía en los programas de estudio de de Educación Cívica de los años 2000, 2005 y 2008 muestra procesos curriculares que afectan, negativamente, la calidad del producto a desarrollar, entre ellas están: los criterios subjetivos con que una persona, asesor nacional, elige los integrantes de la comisión, el trabajo de la comisión se realiza en un lapso de un año con la salvedad de que la selección y la organización de contenidos se realiza entre cinco y seis meses, se omite la sistematización de la experiencia y, por último, la comisión realiza la selección-organización de contenidos sin la creación de criterios explícitos.

Dentro de los criterios implícitamente elaborados por la comisión para seleccionar los contenidos, se encuentran: programas anteriores, teoría curricular, experiencia, formación de destrezas y actitudes en los estudiantes y la pertinencia, para ellos, de los contenidos, la consolidación de Educación Cívica como asignatura y su evaluación en bachillerato, la visión del Ministro y Política del MEP, el contexto histórico, el juicio de expertos, las casas editoriales y un currículum oculto de redes de poder institucional. Por su parte, en relación con la organización, sólo se establecen relaciones con criterios de secuencia y nivel cognitivo del estudiantado.

La anterior circunstancia no sólo impide una estructuración clara y sistemática del elemento político de la ciudadanía, y de la ciudadanía en general, sino además sería una limitante para realizar un abordaje crítico, desde las comisiones, sobre las redes de poder, el proyecto hegemónico, las demandas progresistas y el contexto sociohistórico en general que enmarcan la configuración de la ciudadanía.

En cuanto a los fundamentos curriculares que mediaron en la selección y en la organización de contenidos, se mantiene la constante de pocas referencias explícitas a su utilización, aunque un análisis de los programas permite la determinación de criterios filosóficos, económicos, socioculturales-políticos y psicológicos, en la estructuración del proceso. 
En el caso de los fundamentos filosóficos, se ha podido determinar la influencia de la Ley Fundamental de Educación (en específico los Fines de la Educación Costarricense) y el perfil de salida del estudiante en Tercer Ciclo y Educación Diversificada en el área de Educación Cívica. En relación al fundamento económico, se refleja la transición entre un modelo económico intervencionista, de origen socialdemócrata, y el modelo neoliberal, que a su vez, se entrelaza, dialécticamente, con un fundamento sociocultural y político marcado por una sociedad en discursos de crisis y cuestionamientos a la institucionalidad democrática. Ambos fundamentos influyen notablemente en la configuración de los programas de estudio, ya que estos se estructuran como una respuesta pedagógica a esas demandas del contexto sociohistórico.

Por todo lo anterior, es una imperiosa necesidad la reformulación del proceso de selección y organización de contenidos, y del diseño de los programas de estudio, dentro de un proceso de estructuración y sistematización de sus procesos, con el objetivo de validar sus productos, legitimar sus procesos y perfeccionar sus etapas y, paralelamente, la formulación de pautas que delimiten, con claridad conceptual y pertinencia sociohistórica y crítica, la ciudadanía como construcción sociocultural a desarrollar dentro del sistema educativo formal.

\section{REFERENCIAS}

Apple, Michael. (1997). Política cultural y educación. España: Morata.

Apple, Michael. (1996a). Educación y poder. España: Paidós.

Apple, Michael. (1996b). El conocimiento oficial. La educación democrática en una era conservadora. España: Paidós.

Arias, María, Freer, Sandra, Sole, Aldo y Vásquez, German. (1998). La Ciudadanía Costarricense y el sistema educativo 1920-1949. Tesis para optar por el grado de Licenciatura en Historia. Universidad de Costa Rica. Montes de Oca, San José, Costa Rica.

Asamblea Legislativa de Costa Rica. (2007a). Constitución Política de la República de Costa Rica. Costa Rica: Investigaciones Jurídicas SA.

Aquín, Nora. (2003). En torno a la ciudadanía. En: Aquín, Nora. (Comp). Ensayos sobre la Ciudadanía. Reflexiones desde el Trabajo Social. Argentina: Espacio. 
Cabello, María. (2003). Imaginar e instituir la educación globalizada. En: Jaume Martínez (coord.), Ciudadanía, poder y educación (pp. 35-56). España: GRAO.

Cortina, Adela. (2007). Democracia Deliberativa. En: José Rubio; Ana Salmerón y Manuel Méndez (eds), Ética, ciudadanía y democracia (pp. 32-59). España: Contrastes.

Cortina, Adela. (2001). Ciudadanos del mundo. Hacia una teoría de la ciudadanía. España: Alianza Editorial.

Carretero, Mario. (1993). Constructivismo y educación. Argentina: Aique.

Coll, César. (1991). Psicología y currículum. España: Paidós.

Freire, Paulo. (1997). Educación y participación comunitaria. En: M Castells; R. Flecha; P. Freire; H. Giruoux; D. Macedo y P. Willis, Nuevas perspectivas críticas en educación (pp. 83-96). España: Paidós.

Freire, Paulo. (1990) La naturaleza política de la educación. Cultura, poder y liberación. España: Paidós.

Freire, Paulo. (1984) Pedagogía del Oprimido. España: Siglo XXI.

Gimeno, José. (2003). Volver a leer la educación desde la ciudadanía. En: Jaume Martínez (coord.), Ciudadanía, poder y educación (pp. 11-34). España: GRAO.

Gimeno, José. (2001). Educar y convivir en la cultura global. Las exigencias de la ciudadanía. España: Morata.

Gimeno, José. (1998a). El currículum: una reflexión sobre la práctica. España: Morata. S.A.

Gimeno, José. (1998b). Poderes inestables en educación. España: Morata. S.A.

Gimeno, José. (1997). Pedagogía por objetivos. Obsesión por la eficiencia. España: Morata.

Giner, Salvador. (2007). La dignidad cívica. En: José Rubio; Ana Salmerón y Manuel Méndez (eds), Ética, ciudadanía y democracia (pp. 87-124). España: Contrastes.

Giroux, Henry. (2006) La escuela y la lucha por la ciudadanía. Pedagogía crítica de la época moderna. México: Siglo XXI.

Giroux, Henry; McLaren, Peter. (1998). Sociedad, cultura y educación. España: Miño y Dávila.

Hidalgo, Antonio. (1998). La forzada apertura comercial y el modelo neoliberal de desarrollo en Costa Rica. Revista de Ciencias Sociales, (78-79), 61-74.

Hindes, Barry. (2002). La ciudadanía neoliberal. Revista mexicana de ciencias políticas y sociales, 45, 107-131 
Jiménez, Edgar. (1991). Bases del pensamiento neoliberal. Revista de Ciencias Sociales, (5152), 113-120.

Kemmis, Stephen. (1998). El currículum: más allá de la teoría de la reproducción. España: Morata.

Korten, Alicia. (1997). Ajuste Estructural en Costa Rica. Una medicina amarga. Costa Rica: Departamento de Ecuménico.

Levinson, Bradley; Berumen, Juan. (2007). Educación para la ciudadanía democrática en los países de América Latina: una mirada crítica. Revista Electrónica Iberoamericana sobre calidad, eficacia y cambio en educación, 5 (4), 16-31. Disponible en http://www.rinace.net/arts/vol5num4/art1.pdf.

Lichstenztejn, Samuel. (1988). Políticas económicas neoliberales en América Latina. Revista Centroamericana de economía. 8-9, 125-148

Marshall, Thomas. (1997). Ciudadanía y Clase Social. Revista Española de Investigaciones Sociológicas, 79, 297-344.

Martínez, Jaume (coord.) (2003). Ciudadanía, poder y educación. España: GRAO.

McLaren, Peter. (1997). Pedagogía crítica y cultura depredadora. España: Paidós.

Mejía, Andrés; Perafán, Betsy. (2006). Para acercarse al dragón, para amansarlo, es necesario haberlo amansado primero: una mirada crítica a las competencias ciudadanas. Revista de Estudios Sociales, (23), 23-35.

Ministerio de Educación Pública. (2008). Programas de Estudio Educación Cívica. Tercer Ciclo y Educación Diversificada. Costa Rica: ICER.

Ministerio de Educación Pública. (2005). Programas de Estudio Educación Cívica. Tercer Ciclo y Educación Diversificada. Costa Rica: s.e.

Ministerio de Educación Pública. (2000). Programas de Estudio Educación Cívica. Tercer Ciclo y Educación Diversificada. Costa Rica: s.e.

Miranda, Guillermo. (2007). Política curricular, crisis de legitimación y hegemonía neoliberal. Una visión desde la sociología de la educación critica. Revista de Ciencias Sociales, (115), 13-34.

Miranda, Guillermo. (2001). Capitalismo global y cambio educativo. Los fundamentos estructurales e ideológicos de la política educativa neoliberal. Revista Educare, 4 (7), 1342. 
Miras, Mariana. (2002). Un punto de partida para el aprendizaje de nuevos contenidos: los conocimientos previos. En: C. Coll; E. Martín; T. Mauri; J. Onrubia; J. Solé y A. Zabala, EI constructivismo en el aula (pp. 47-64). España: GRAO.

Molina, Iván. (2002). Costarricense por dicha. Identidad nacional y cambio cultural en Costa Rica durante el siglo XIX y XX. Costa Rica: EUCR.

Molina, Iván y Palmer, Steven. (2000). Educando a Costa Rica: alfabetización popular, formación docente y género (1880-1950). Costa Rica: Porvenir.

Ochman, Marta. (2006). La reconfiguración de la ciudadanía. Los retos del globalismo y de la posmodernidad. México: Tecnológico de Monterrey.

Palacios, María. (2006a). Enseñanza Cívica en el periodo oligárquico liberal en Costa Rica, 1886-1920: un acercamiento desde la teoría de género y de la educación cívica. Actualidades Investigativas en Educación, 6 (1), 1-21. Disponible en http://revista.inie.ucr.ac.cr/articulos/1-2006/civica.php.

Palacios, María (2006b). La enseñanza de la instrucción cívica costarricense: un acercamiento desde el contenido de los programas educativos en el periodo: 1886-1920. Actualidades Investigativas en Educación, 6 (3), 1-30. Disponible en http://revista.inie.ucr.ac.cr/articulos/3-2006/civica.php.

Pérez, María. (1992). Entre lo escolar y los medios informativos: políticas neoliberales y educación. Ciencias Sociales, (57), 41-55.

Pérez, María y González, Yamileth. (1991). De la crisis financiera al proyecto neoliberal: la educación superior en Costa Rica. Káñina, XV (1-2), 341-348.

Piñuel José Luís. (2002) Epistemología, metodología y técnicas del análisis de contenido. Estudios de Sociolingüística 3 (1), 1-42

Sandín, María Paz. (2003). Investigación Cualitativa en Educación. Fundamentos y tradiciones. España: Mc Graw Hill.

Solano, Edgar. (2006) ¿Enseñamos Estudios Sociales o Historia? Actualidades Investigativas en Educación, 6 (2), 1-21. Disponible en http://revista.inie.ucr.ac.cr/articulos/22006/historia.php.

Tamayo, Juan. (2003). Ética liberadora del cristianismo frente a teología neoliberal del mercado. Senderos, 25, 743-795

Torres, Jurjo. (2001). Educación en tiempos del Neoliberalismo. España: Morata S.A.

Torres, Jurjo. (1998). El currículum oculto. España: Morata. 
Toruño, César. (2010). Los fundamentos curriculares de la ciudadanía en un Estado Neoliberal: el caso del sistema educativo costarricense. Actualidades Investigativas en Educación, 10 (2), 1-25. Disponible en http://revista.inie.ucr.ac.cr/articulos/2-2010/ciudadania.php.

Tyler, Ralph. (1973). Principios básicos del currículum. Argentina: Troquel.

Vargas, Luis. (2002). Costa Rica, 1985-1997: liberalización y ajuste estructural o la autodestrucción del neoliberalismo. Costa Rica: EUNED.

Vargas, Luis. (2001). Costa Rica hoy: Una sociedad en crisis. Costa Rica: EUNED. 